\title{
Anabases
}

ANABASES Traditions et réceptions de l'Antiquité

19 | 2014

Varia

\section{La réception de Franz Cumont : à propos de quelques publications récentes / II}

Constantinos Macris

\section{OpenEdition}

1 Journals

Édition électronique

URL : http://journals.openedition.org/anabases/4698

DOI : 10.4000/anabases.4698

ISSN : 2256-9421

Éditeur

E.R.A.S.M.E.

Édition imprimée

Date de publication : 1 avril 2014

Pagination : 251-278

ISSN : 1774-4296

\section{Référence électronique}

Constantinos Macris, "La réception de Franz Cumont : à propos de quelques publications récentes / I », Anabases [En ligne], 19 | 2014, mis en ligne le 01 avril 2017, consulté le 21 octobre 2019. URL http://journals.openedition.org/anabases/4698; DOI : 10.4000/anabases.4698 
Anabases 19 (2014), p. 251-278

\section{La réception de Franz Cumont : à propos de quelques publications récentes / II}

CONSTANTinos Macris

Franz Cumont, Lux perpetua, volume édité par Bruno Rochette \& André Motte avec la collaboration de Bastien Toune, Academia Belgica - Institut historique belge de Rome / Torino : Nino Aragno Editore, 2009 (coll. « Bibliotheca Cumontiana. Scripta maiora ", 2). Pp. CXLIX - 595 (broché). ISBN 978-88-8419-423-7. €72.00 (diffusion Brepols Publishers).

Corinne Bonnet, Carlo Ossola, John Scheid (dir.), Rome et ses religions : culte, morale, spiritualité. En relisant Lux perpetua de Franz Cumont [Journée d'étude au Collège de France, 31 mars 2010], Palermo - Caltanissetta : Salvatore Sciascia Editore, 2010 (coll. "Supplemento a Mythos. Rivista di Storia delle Religioni » [n.s.], 1). Pp. IX - 145 (broché). ISBN 978-88-8241-355-2. €20.00

Au déBut du PRINTEMPS de 1943, en pleine occupation allemande de Paris, Franz Cumont, alors âgé de 75 ans, vient présenter au public français la primeur de ses dernières recherches à l'invitation du Collège de France, et dans le cadre des conférences de la Fondation Michonis : elles portent sur L'évolution de l'idée d'immortalité dans le monde romain ${ }^{1}$. Presque 40 ans s'étaient alors écoulés depuis la première invitation de la même institution prestigieuse, qui avait honoré le grand savant belge en lui

1 Apparemment à la même occasion, Cumont donna aussi une conférence à l'École normale supérieure sur «L'astrologie et les morts prématurés », un sujet relevant également de la 
donnant l'occasion de prononcer, en 1905, les conférences publiques qui ont abouti à ce qui fut par la suite son ouvrage le plus célèbre et celui qui a eu la plus large audience à travers le monde entier : Les religions orientales dans le paganisme romain $[R O]^{2}$. Les conférences de 1943, quant à elles, ne seront publiées que bien après la fin de la guerre, en 1949, par les soins de Louis Canet et de la marquise de Maillé : ce sera Lux perpetua $[L P]$, l'ouvrage ultime, et posthume, de Cumont [C.], auquel il prodiguait encore ses soins jusqu'aux derniers jours de son existence, une sorte de testament scientifique, intellectuel et spirituel, à la fois monument d'érudition inégalée, destiné à rester $x \tau \tilde{\eta} \mu \alpha$ غ̇ $\varsigma$ ảé́, et message d'espoir adressé à une humanité qui, après deux guerres mondiales absolument terrifiantes et ravageuses, sortait profondément traumatisée.

C'est donc avec raison, me semble-t-il, que la Bibliotheca Cumontiana poursuit la réédition des Scripta maiora de C. avec cet opus magnum qui, par un effet de symétrie ou de composition annulaire, clôt le cycle des grandes synthèses à destination du public cultivé, qui avait été ouvert avec les $R O$ : ainsi, la boucle étant en quelque sorte bouclée, l'historiographie moderne est en mesure non seulement d'étudier la genèse de $L P$ en la situant dans le contexte des débats intellectuels et des orientations scientifiques majeures de son temps, mais aussi de s'offrir une vision rétrospective de l'œuvre cumontienne dans son ensemble. Une telle vision permet de mettre en valeur les idées maitresses, les Leitmotive, les constantes, voire les obsessions ayant marqué la pensée de l'auteur, mais aussi de mesurer le chemin parcouru par lui depuis les débuts de sa carrière, en repérant les approfondissements successifs et les précisions apportées sur des points particuliers, les zones d'ombre ou de doute, les moments (relativement rares) de révision ou de retractatio, ou alors ceux d'une inspiration nouvelle ayant surgi au contact de documents inédits ou en réaction, positive ou négative, à des interprétations novatrices et hardies de ses contemporains.

De tels questionnements sont en effet au cœur des préoccupations de la très riche Introduction historiographique, longue de plus de 110 pages (p. xxxiii-cxlix), qui sert de companion au lecteur de $L P$, co-écrite par Bruno Rochette et André Motte, de l'université de Liège. Le travail de réflexion peut être poursuivi et prolongé, de manière féconde, et polyphonique, dans un supplément de la revue Mythos. Rivista di Storia delle Religioni de l'université de Palerme, qui, depuis un moment, mène une nouvelle vie. Ce volume dirigé par Corinne Bonnet, Carlo Ossola et John Scheid constitue en réalité les Actes [cité Actes dans la suite du texte] d'une journée d'étude que ses organisateurs avaient intitulée, de manière intentionnellement intrigante, "Franz Cumont revient au Collège de France ", faisant ainsi allusion au come back posthume de C. à Paris, par le biais de la réédition de $L P$, après les deux séries de conférences qu'il y

thématique de la vie après la mort, auquel sera consacré le chap. VII de LP (cf. p. 355, n. 1). - Sur la Fondation Michonis, voir S. Rey dans Actes, p. 21, n. 1.

2 Sur la réédition récente des $R O$, voir la discussion parue dans le numéro précédent d'Anabases (18 [2013], p. 215-226). 
avait données en 1905 et 1943 . Publiées tout juste un an après la parution de cette «nouvelle» $L P$, les dix contributions réunies dans ce petit volume (ca 150 p.) joliment édité se proposent, à en croire le sous-titre, d'offrir une (ou plutôt des) "relecture(s) » plurielle(s) de l'ouvrage de $1949^{3}$, plus de soixante ans après. L'examen conjoint de ces études et de l'Introduction historiographique de LP permet donc de mettre l'ouvrage en perspective, et de ce fait constitue une excellente base à partir de laquelle chacun peut réfléchir sur le sens et les présupposés explicites ou implicites de ce " grand livre sur un grand sujet » (pour paraphraser C. ...), sur sa postérité et, surtout, sur ce qu'il peut encore signifier pour nous en ce début $\mathrm{du} \mathrm{XXI}^{\mathrm{e}}$ siècle, et ce que sa méthodologie, son orientation générale, ses impasses mêmes, peuvent inspirer à la jeune génération de chercheurs spécialisés dans l'étude des phénomènes religieux de l'Antiquité dans leur triple dimension : cultuelle-rituelle, morale et spirituelle (reflétée dans la triade « culte, morale, spiritualité " évoquée pertinemment dans le titre des Actes).

Mais il serait sans doute plus approprié de commencer, ne serait-ce qu'à titre de simple rappel, par la présentation du livre de C. en tant que tel - sa table des matières, sa structure générale, son fil directeur, son titre, les thèses principales qui y sont étayées, le message qu'il veut passer au lecteur ; la méthode employée, le type de sources exploitées, l'usage de la bibliographie -, avant d'essayer d'en situer la genèse, successivement mais corrélativement, d'un point de vue " interne " puis « externe ». Il s'agirait, d'une part, d'examiner le rapport que cette genèse entretient (a) à l'œuvre antérieure de C. et aux questions qui, de manière permanente, la traversent de part en part et (b) aux circonstances humaines de la vie de l'auteur dans lesquelles elle est ancrée ; d'autre part, de reconstituer le contexte scientifique dans lequel elle a pu émerger - contexte forcément international, étant donné l'envergure de la personnalité scientifique de C., les filiations multiples de sa pensée et son immense érudition. C'est la tâche à laquelle s'est attelé $\mathrm{Br}$. Rochette ( $L P$, p. xxxiv-lxxxvi), quoiqu'en abordant les points susmentionnés dans un ordre différent.

Au niveau descriptif, il serait fastidieux de s'attarder sur des détails, surtout si l'on tient compte du fait que C. lui-même, en suivant une pratique qui n'est plus courante à notre époque, avait pris le soin de confectionner une table des matières très détaillée, vraiment impressionnante $(25$ p.), dont la lecture rend parfaitement clair et immédiatement saisissable le fil directeur de sa pensée, jusqu'à ses moindres détours (p. vii-xxxii ; cf. aussi 2.1. Structure, p. lvi-lxii et la récapitulation finale, p. 441-442). Car, en effet, cet ouvrage généreux et magistralement synthétique qui, en dépit de sa nette focalisation sur le monde hellénistique et surtout l'empire gréco-romain jusqu'au

3 "En relisant Lux perpetua. Franz Cumont et les savants de son temps " est d'ailleurs le titre d'une étude d'André Mотте publiée dans le vol. 111 (1999) des Mélanges de l'École française de Rome (Italie et Méditerranée), consacré à Franz Cumont et la science de son temps (éd. A. Rousselle), aux p. 507-524. Le choix de la même formule pour le sous-titre des Actes est sans doute une forme d'hommage indirect, mais perceptible, à ce qui s'avère être une des premières études à caractère historiographique consacrées à $L P$. 
" triomphe du christianisme ", embrasse en réalité l'évolution des idées antiques relatives à la mort, à la vie future et à l'au-delà dans la diachronie - dans la « longue durée " -, depuis Homère voire les origines indo-européennes, ou même la préhistoire, jusqu'au néoplatonisme tardif, avec des prolongements dans le christianisme antique, médiéval et moderne, ou encore dans les «survivances » du folklore contemporain, cet ouvrage donc est bel et bien doté d'un fil rouge ${ }^{4}$ qui lui confere une structure linéaire " ascensionnelle " : il s'agit (pour reprendre la formulation adoptée dans la présentation du livre figurant sur la jaquette) de montrer « comment, au fil des siècles, les Enfers se sont déplacés des entrailles de la terre, où les plaçait la tradition littéraire grecque, vers le firmament du ciel, pour donner finalement naissance au thème de l'eschatologie céleste »- lunaire, solaire ou stellaire. C'est à la luminosité éclatante liée à cette nouvelle perspective eschatologique, à la lumière en tant que symbole d'immortalité, que semble faire allusion en premier lieu la "lumière éternelle " du titre - un titre intentionnellement mystérieux et polysémique par ailleurs, ouvert à plusieurs interprétations, y compris christianisantes (voir p. 1-liii).

Un autre élément structurant de l'approche globale de C., qui semble aller à l'encontre du précédent, mais qui finalement se croise et s'entrelace avec lui comme les fils de trame et les fils de chaîne d'un tissu, est le mouvement dialectique de type hégélien qui va des "vieilles croyances" (populaires) [thèse] à la réaction de la " critique philosophique " (de type rationaliste/ sceptique et/ou matérialiste/ épicurien) [antithèse], avant d'arriver à la nouvelle doctrine, celle de l'immortalité céleste [synthèse]. C'est évidemment une schématisation qui, de nos jours, prête facilement le flanc à la critique, mais à laquelle adhéraient encore tout aussi naturellement les plus grands esprits scien-

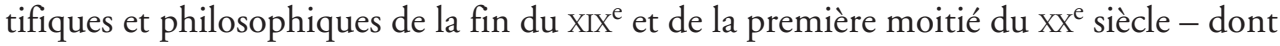
par exemple E. Zeller, l'éminent historien de la philosophie grecque - dans leur effort de donner un sens dynamique au cours de l'histoire des idées. Et il en va de même de l'idée évolutionniste du progrès de l'humanité qui sous-tend la structure linéaire de $L P$ évoquée plus haut ; car, pour reprendre (en le lisant un peu différemment) un slogan publicitaire récent, « le progrès, il faut y croire pour le voir » ... surtout après les catastrophes des deux guerres mondiales du siècle précédent...

Au-delà des idées directrices et des éléments structurants que l'on vient de relever, il ne faut pas oublier que $L P$ véhicule également des thèses interprétatives fortes. On pourrait les résumer sous trois chefs : (1) la doctrine de l'ascension de l'âme est d'origine iranienne ; par l'intermédiaire des Mages d'Asie Mineure, les "Chaldéens », elle

4 Contrairement à un ouvrage comme celui de Carlo PASCAL, par exemple (Le credenze d'oltretomba nelle opere letterarie dell'Antichità classica, 2 vol., Catania 1912, 2e éd. 1923 [réimpr. L'oltretomba dei pagani, 2 vol., Genova 1981-1985]), ouvrage de référence qui reste à un niveau purement descriptif (Cumont doit l'avoir beaucoup utilisé, puisqu'il l'avait inclus dans sa liste d'ouvrages cités en abréviation dans les notes).

5 Il s'agit en réalité d'une thèse empruntée à W. Bousset ; voir $L P$, p. lxxxiii-lxxxiv, et cf. infra, p. 256. 
a été transmise aux Pythagoriciens, qui en ont assuré la diffusion dans le monde grec, puis romain ; Platon, l'ancienne Académie et le platonisme ont pris le relais par la suite et ont contribué de manière décisive aux développements ultérieurs ; (2) l'immortalité astrale ne relève pas de croyances populaires, mais présuppose un système scientifique de cosmologie et d'astronomie-astrologie ; (3) dans le développement de cette doctrine, la philosophie d'une part, et en premier lieu la tradition pythagoricienne et platonicienne, et les mystères d'autre part, notamment ceux venus d'Orient, ont joué un rôle capital en y introduisant une forte composante morale. Voilà trois thèses que l'on ne serait sans doute pas prêt à accepter aujourd'hui telles quelles, mais qui constituent tout de même des hypothèses fécondes dont la reprise à nouveaux frais donnerait sans doute des résultats intéressants.

Le spécialiste de C. remarquera que ces thèses sont aussi très étroitement liées à certaines constantes (on pourrait même dire "idées fixes") de la pensée de C., surtout en ce qui concerne l'importance accordée à l'influence de l'Orient sur les idées religieuses et philosophiques du monde grec, puis romain, à l'astrologie, à la philosophie, au pythagorisme, aux processus de moralisation, intellectualisation et spiritualisation de la religion - l'horizon d'attente étant pour lui, depuis le début, l'explication des conditions et des causes qui ont ouvert la voie au " triomphe du christianisme " sur le paganisme romain. Cela ressort clairement de l'examen de LP en tant qu'auvre récapitulative de la pensée de C., en tant que synthèse des résultats de recherches de toute une vie (p. xlii-xlix). Cet examen est mené à l'intérieur d'un développement capital de l'Introduction historiographique consacré à l'étude de la "genèse " de LP (xxxiv-lvi) : (a) son ancrage dans l'histoire (douleur collective à l'issue des deux guerres mondiales) et dans la vie de l'auteur (qui, au seuil de ses 80 ans, médite sur la mort qui s'approche et sur l'au-delà) ; puis (b) le rapport "génétique " que $L P$ entretient avec une synthèse antérieure, plus " exploratoire ", de lui sur le même sujet : After Life in Roman Paganism (1923), et avec un travail plus ciblé, plus concret, et plus récent, portant, comme son travail monumental de jeunesse sur Mithra (les fameux TMMM), sur des monuments figurés tels qu'ils sont éclairés par les inscriptions et les textes littéraires : Recherches sur le symbolisme funéraire des Romains (1942). Br. Rochette y met en évidence le cheminement intellectuel de C., au fil d'une série d'articles et d'ouvrages monographiques, en permettant de suivre de près l'évolution de sa pensée. Grâce à cette analyse le lecteur se rend compte en même temps à quel point l'étude de $L P$ peut constituer un excellent point de départ pour connaître, mutatis mutandis, l'œuvre de C. dans son ensemble.

Si cette perspective peut être accueillie comme un cadeau insoupçonné, en revanche une grande part des attentes qu'un lecteur averti pourrait avoir de cette Introduction historiographique se concentre tout naturellement sur la proposition de contextualisation intellectuelle de $L P$ par rapport aux courants de recherche et aux réalisations scientifiques de la fin du XIX et de la première moitié $\mathrm{du} \mathrm{Xx}$ siècle - surtout si l'on connaît déjà la synthèse analogue, très féconde, proposée en introduction aux $R O$ rééditées en 2006. L'Introduction a effectivement veillé à satisfaire ces attentes légitimes, en essayant surtout de situer $L P$ par rapport à deux milieux, et deux séries de travaux, 
avec lesquels C. était particulièrement familier : les études sur l'au-delà issues de la Religionsgeschichtliche Schule et celles, écrites en français, sur le pythagorisme (p. lxxxlxxxvi). Br. Rochette montre d'abord à quel point le savant belge avait très tôt assimilé les résultats des recherches exposées dans trois ouvrages allemands fondamentaux : (1) la Nekyia d'A. Dieterich (1893), sur les origines (purement grecques selon lui - ce qui n'était pas propre à séduire C. dont l'" orientalisme » est bien connu...) des croyances eschatologiques de la fin de l'Antiquité ; (2) le très long article (77 p.) de W. Bousset sur Die Himmelsreise der Seele (1901), dont C. partagera avec enthousiasme la thèse principale sur l'origine mazdéenne-zoroastrienne (et non pas grecque ni babylonienne) de la doctrine de l'ascension de l'âme ; et (3) l'étude volumineuse de J. Kroll intitulée Gott und Hölle. Der Mythos vom Descensuskampfe (1932), qui introduisit une idée nouvelle : "Que les ascensions célestes ne représenteraient qu'une catabase à rebours ", et qu' "un descensus incognitus est suivi par un ascensus gloriosus d'un sauveur " (p. lxxxv) ${ }^{6}$. Sans oublier que le paradigme suivi par C. pour l'ensemble de LP est bien celui de la magnifique Psyché d'E. Rohde (p. liv-lvi ${ }^{7}$ ). Le panorama de l'influence de l'Altertumswissenschaft allemande sur $L P$ aurait pu être enrichi davantage avec une discussion de l'apport du commentaire magistral d'Ed. Norden au livre VI de l'Énéide virgilienne (noté p. xl). On aurait aimé voir également ici ne serait-ce qu'une brève mention des travaux capitaux des spécialistes d'astronomie, de cosmologie et de croyances astrologiques qui constituent le complément nécessaire de l'étude de l'ascension céleste et de l'immortalité astrale menée dans LP: P. Capelle, De luna stellis lacteo orbe animarum sedibus (Diss. Halle 1917) ; Fr. Boll, Sphaera (Leipzig 1903), ainsi que de nombreux articles du même auteur réunis en 1950 sous le titre Kleine Schriften zur Sternkunde des Altertums; E. Pfeiffer, Studien zum antiken Sternglauben (Leipzig 1916).

Côté français (ou plus exactement francophone, étant donné qu'A. Delatte était belge et G. Méautis suisse), sont relevés à juste titre Les travaux français sur les Pythagoriciens (p. lxxxiv-lxxxvi) - étant donné le rôle central reconnu au pythagorisme dans les analyses de $L P$-, avec notamment L'origine astronomique de la croyance pythagoricienne en l'immortalité céleste de L. Rougier (1933), un ouvrage que C. appréciait, mais dont il ne partageait pas la thèse d'une origine grecque, et scientifique, de l'idée

6 Les éditeurs ont omis de noter ici que Cumont avait rédigé un compte rendu extrêmement positif de cet ouvrage (paru dans la Revue belge de philologie et d'histoire 12 [1933], p. 264-265), où il relevait notamment le soin avec lequel Kroll avait étudié le motif de " la lutte des dieux contre les puissances du monde souterrain " dans plusieurs civilisations de l'Orient, avant de souligner que "[d]epuis l'époque hellénistique, des conceptions empruntées aux vieilles religions de l'Asie pénétrèrent la mythologie hellénique et les Grecs, en les adoptant, les transformèrent, les remodelèrent, leur donnèrent la forme sous laquelle elles devaient se perpétuer désormais ».

$7 \quad$ Il aurait été plus pertinent, me semble-t-il, que ces pages figurent dans le contexte immédiat de la section consacrée à L'environnement scientifique contemporain, et non pas à trente pages de distance. 
de l'ascension de l'âme vers le ciel, étant convaincu lui-même que cette idée était bien antérieure, et de provenance orientale (iranienne) et religieuse (voir p. lxxxv, n. 297). Mais là aussi, on aurait attendu un panorama plus complet : par exemple, une mention explicite des Études sur le Songe de Scipion de P. Boyancé $(1936)^{8}$, ouvrage qui figure dans la liste des " principales abréviations employées dans les notes " établie par C. luimême (p. 27), et dont non seulement la thématique mais aussi le recours à des sources pythagoriciennes dans l'analyse sont capitaux pour le propos de $L P$; ou encore des références au livre de J. Carcopino sur La basilique pythagoricienne de la Porte Majeure (1926) ou au chapitre sur "Le pythagorisme primitif et ses relations avec l'Orient" d'Éos ou Platon et l'Orient (1945) de Joseph Bidez l'ami et complice de C. pour les Mages hellénisés - ouvrages si souvent utilisés tous les deux qu'ils figurent également dans les "abréviations " de LP. Quant à Isidore Lévy, dont les deux ouvrages sur la "légende de Pythagore" " sont bien sûr utilisés par C. dans $L P$, il est important de souligner la prudence de la position de notre auteur face aux théories totalement spéculatives et parfois même farfelues de Lévy sur la " descente aux enfers » de Pythagore qui serait l'un des principaux thèmes du dialogue Abaris d'Héraclide le Pontique, véritable Divina commedia ayant servi de source d'inspiration et de modèle pour les représentations grecques (Lucien), romaines (Virgile), juives, et même égyptiennes (récit démotique de Siosiri) de l'Hadès. C. n'en tient tout simplement pas compte pour ses propres reconstructions ${ }^{10}$.

Au-delà de l'intérêt proprement historiographique que présente $L P$, cet ouvrage reste surtout, aujourd'hui encore, une véritable somme reflétant l'état des connaissances et de la recherche sur le sujet au milieu du Xx siècle. Cela est évident à chaque page, mais il saute surtout aux yeux, de manière éclatante, à la lecture de ces "véritables joyaux d'érudition " (p. lxv) que sont les dossiers, portant sur des sujets très variés, constitués par C. dans les «notes complémentaires» (p. 443-480 ${ }^{11}$ ), auxquelles il avait donné tous ses soins. Br. Rochette le montre bien aussi dans la section consacrée aux sources littéraires en grec et latin, aux inscriptions et aux papyrus utilisés par C. pour étayer ses analyses dans $L P$ (p. lxiv-lxxx). (Dans cette section, soit dit en passant, on peut apprécier le louable effort qui a été fait, dans les notes de bas de page, pour fournir au lecteur d'aujourd'hui toutes les références nécessaires à des études plus récentes sur ces sources. Un Appendice bibliographique [p. cxliv-cxlviii] extrêmement utile est ajouté également à la fin de l'Introduction ${ }^{12}$ ).

8 Un titre qui manque aussi aux p. lxvii-lxviii, où il est question du Songe.

9 I. LÉvy, Recherches sur les sources de la légende de Pythagore, Paris 1926 [réimpr. New York 1987] ; ID., La légende de Pythagore, de Grèce en Palestine, Paris 1927.

10 Voir LP, p. 97 sq. et 455-456 (note complémentaire sur les Descentes aux Enfers), ainsi que l'index, s.v. "Héraclide Pontique ».

11 Les «notes complémentaires » des p. 480-543, en revanche, sont dues à L. Canet.

12 Pour d'autres compléments possibles, voir la liste d'addenda proposée ici en fin d'article. 
Un autre aspect qui rend la $L P$ exceptionnelle en lui donnant une "épaisseur " existentielle rarement rencontrée dans des ouvrages appartenant à la catégorie " monuments d'érudition ", est son rapport intime avec la vie de C. Ici il ne serait pas exagéré de dire que l'écriture et la méditation sur la condition mortelle de l'homme et sur la perspective d'un au-delà radieux telle qu'elle a été envisagée dans de nombreuses traditions antiques a certainement joué pendant les dernières années du vieux C. un rôle d'exercice spirituel - un peu à la manière des Eis $\dot{\varepsilon} \alpha v \tau o ́ v$ de Marc Aurèle ${ }^{13}$. En même temps, la "lumière éternelle » du titre représente un message d'espoir à la fois conjoncturel (adressé à une Europe tout juste sortie de la Seconde Guerre mondiale ${ }^{14}$ ) et universel (adressé à l'humanité tout entière).

Une des questions soulevées par ce "message " est de savoir dans quelle mesure il serait susceptible d'une interprétation catholique. Les éditeurs de la nouvelle $L P$ soulignent à juste titre le rôle joué dans ce sens par la notice introductive de Louis Canet, qui semble avoir voulu « christianiser » de force l'image du dernier C. en insistant sur sa mort chrétienne, ou à tout le moins transformer $L P$ en un plaidoyer pour la croyance en l'immortalité (voir n. 491 in fine). Ce sont là des manipulations qui ne sont pas toujours passées inaperçues, mais qui peuvent avoir influencé la réception de LP non seulement parmi les spécialistes de la religion et de la philosophie grecque et romaine, mais aussi parmi les lecteurs cultivés d'orientation laïque athée, de plus en plus nombreux dans le monde francophone de l'après-guerre. Dans sa contribution aux Actes (voir infra, p. xxx), Corinne Bonnet remet en doute cette présentation " discutable, voire tendancieuse " de C. en rappelant que Canet lui attribue par ailleurs un patriotisme belge et un anti-germanisme dans lesquels $C$. ne se reconnaitrait sans doute pas. En abondant dans son sens, on devrait souligner aussi à quel point plusieurs des notes additionnelles rédigées par Canet insistent un peu lourdement sur des éléments chrétiens, et ont une orientation nettement différente de celles provenant de la main de C. ${ }^{15}$ - notamment la très longue (p. 523-543) et significative N.C. XXXV, Immortalité et résurrection, sur laquelle se clôt, comme par hasard, l'ouvrage...

Dans la deuxième partie de l'Introduction (p. lxxxvi-xclviii), due à André Motte, on passe à l'étude détaillée de la réception immédiate et de la postérité de $L P$ jusqu'à

13 Voir P. Нadot, La citadelle intérieure : une introduction aux "Pensées " de Marc Aurèle, Paris $1997^{2}$ [ $1^{\text {re }}$ éd. 1992 ; réimpr. 2005 en "Livre de poche ", avec les deux parties du titre inversées]. - Il est peut-être révélateur à cet égard que C. ait écrit des passages particulièrement empathiques sur l'empereur philosophe : voir LP, p. 154-155 (repéré et souligné par Canet dans sa préface, p. 22-23).

14 Il n'a pas échappé aux spécialistes de C. que son After Life a été rédigé et publié également après la fin de la Première Guerre mondiale. L'Introduction émouvante de LP reprend même littéralement des passages entiers de celle de l'ouvrage de 1922, que la Seconde Guerre mondiale avait malheureusement rendus de nouveau actuels.

15 Voir en particulier la N.C. XXVIII, sur Éros et Agapè ; XXIX, sur la Vision béatifique; XXX, sur les Parentalia chez les Chrétiens. 
nos jours, destinée à mettre en lumière la taille de l'audience de l'ouvrage, l'accueil qui lui a été réservé par la critique, les évaluations positives et négatives dont il a fait l'objet et le degré de sa présence effective dans les études et les débats ultérieurs. Pour ce faire, l'auteur examine dans un premier temps les réactions des spécialistes au moment de la parution de $L P$, grâce à un survol des recensions parues dans les revues savantes ou dans des périodiques d'intérêt général, ainsi que des hommages rendus lors du décès de $\mathrm{C}$., dans lesquels ses disciples et ses amis ont annoncé son ouvre posthume en s'attardant quelque peu sur son contenu. Dans un second temps, il analyse les données statistiques fournies par le dépouillement des notices bio-bibliographiques de C. parues dans des ouvrages à caractère encyclopédique, des manuels généralistes de religion grecque et romaine et des études spécialisées portant sur l'afterlife (p. cvi-cxlviii). La question qui anime cette enquête nécessaire - mais qui par endroits s'avère à vrai dire un peu trop longue et fastidieuse - semble être : est-ce que la $L P$ est sortie indemne de l'épreuve terrible du Temps ? Est-ce qu'on peut la considérer véritablement comme un " grand classique ", à l'instar de la Psyché de Rohde par exemple ? Et peut-être aussi indirectement : est-ce que sa réédition aujourd'hui est justifiée et répond à une demande réelle du monde savant et/ou du grand public?

La réponse aux deux premières questions semble assez embarrassante : non ! LP a connu en réalité " une audience somme toute assez limitée ». Mais cela n'enlève franchement rien ni aux qualités exceptionnelles de l'ouvrage ni à l'intérêt qu'il a pour nous aujourd'hui, et surtout n'invalide point la très louable initiative de réédition de $L P$ - tant il est vrai que cet ouvrage est non seulement une somme d'érudition de valeur inestimable depuis longtemps introuvable en librairie, mais aussi, comme le dit si bien C. Bonnet (Actes, p. viii), " une référence incontournable et féconde ", un "monument de science et de littérature " qui " n’a pas véritablement été remplacé, tant le spectre de questions qu'il aborde est large et défie nos compétences plus sectorielles d'aujourd'hui ». Ayant admirablement assimilé et intégré les acquis des sciences de l'Antiquité relatifs au sujet qu'il aborde jusqu'en 1947, ce livre " garde, intact, le charme des grandes synthèses reposant sur une connaissance intime de l'ensemble des sources antiques et usant avec une élégance parfaite du style de la grande littérature scientifique du milieu du $x^{\mathrm{e}}$ siècle » - celle qui, rappelons-le, était même digne de recevoir le prix Nobel de littérature, comme ce fut le cas au tout début du même siècle avec Theodor Mommsen en 1902.

Cela dit, le rapport de LP avec la Psyché d'E. Rohde et avec l'After Life de C. luimême mérite qu'on s'y arrête un peu. Pour l'Antiquité grecque, le grand classique sur les conceptions de la vie post-mortem est incontestablement la Psyché. Dès 1922, C. remarquait dans After Life (p. xi) : "But we do not possess for the Roman imperial epoch a counterpart to Rohde's classical volume, Psyche, for the earlier Greek period, that is, a work in which the whole evolution of Roman belief and speculation regarding a future life is set forth. These lectures cannot claim to fill this gap. They may however be looked upon as a sketch of the desired investigation, in which, though without the detailed citation of supporting evidence, an attempt at least has been made to trace the broad outlines of the 
subject in all its magnitude ". En se lançant, jusqu'à la fin de sa vie, dans cet ongoing project que fut la rédaction de $L P$, C. se situait donc, aussi discrètement soit-il, dans un rapport agonistique avec Rohde, en nourrissant sans doute l'ambition de l'égaler en proposant un pendant romain de Psyché qui prendrait le relais pour la période impériale. Ainsi, avec leurs titres lapidaires, l'un en grec, l'autre en latin, Psyché et Lux perpetua deviendraient deux ouvrages jumeaux, emblématiques en matière de conceptions de l'âme et de l'au-delà, abordant en deux temps, l'un, l'« invention " de l'âme immortelle séparée du corps à l'époque archaïque, l'autre, sa lente mais ferme remontée vers la lumière éternelle de l'immortalité céleste à partir surtout de l'ère hellénistique.

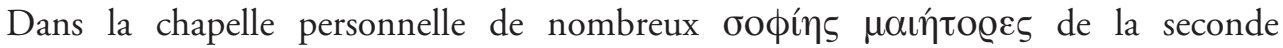
moitié $\mathrm{du} \mathrm{xx}^{\mathrm{e}}$ siècle il en fut probablement ainsi. Mais comme on l'a déjà dit plus haut, les indications recueillies patiemment par A. Motte donnent une image tout à fait différente de la postérité de $L P$ - et ce en dépit d'un accueil globalement très positif au moment de sa sortie en librairie. Pour le dire platement, elles illustrent, de manière inattendue, le relatif insuccès de $L P$, qui, contrairement à la Psyché, ne connut aucune traduction en langue étrangère et seulement deux impressions très confidentielles (en 1976 et 1987), et qui n’a pas réussi à s'imposer durablement à la discipline : dans les manuels de religion antique par exemple, le constat un peu amer est qu'après $1980 \mathrm{LP}$ subit une éclipse totale en religion grecque et quasi-totale en religion romaine.

Plus étonnant encore, l'After Life que, dès sa parution, C. considérait comme une simple esquisse, ou une ébauche, des recherches qui restaient encore à faire dans ce domaine, semble avoir été lu beaucoup plus que $L P$ (peut-être pour des raisons purement linguistiques ?), et il lui a fait de l'ombre - C. ne l'avait-il pas désignée lui-même comme " un After Life français " ? (même s'il s'était empressé de préciser aussitôt : "fortement remanié et amplifié " [cf. p. xxxv] - ce qui est absolument vrai). Ainsi, d'une manière incompréhensible et vraiment injuste, les avancées réalisées par $L P$ ont été largement ignorées. Elles sont pourtant considérables. C. ne s'y est pas contenté d'une simple mise à jour, vu le quart de siècle qui s'était écoulé entre 1922 et 1947 - même s'il a tenu compte, très soigneusement, à la fois de ses travaux personnels en la matière et des travaux publiés par ses collègues, comme le montre la liste des abréviations établie par lui aux p. 27-30, où il est évident que la plupart des travaux ayant accompagné C. constamment dans ses réflexions et approfondissements successifs ont une date de publication postérieure à 1922. Il a changé complètement la conception de l'ensemble en disposant les chapitres dans un ordre différent, et entièrement refondu le chapitre sur L'astrologie et les morts prématurés (355-396) ${ }^{16}$. Il y a surtout ajouté plusieurs sections nouvelles, importantes aussi bien du point de vue du contenu que sur le plan quantitatif: Offrandes funéraires (p. 59-87) ; Fantômes et nécromants (112-144);

16 Pour les publications antérieures de Cumont sur ce même thème, voir R. LANE Fox dans les Actes (p. 83-96) - qui ne signale pourtant pas le chap. V, "Untimely death », d'After Life. 
Les mystères (279-322 : une récapitulation, évidemment, des $R O$ ). Il a intégré les acquis de son autre ouvrage majeur, les Recherches sur le symbolisme funéraire des Romains (Paris 1942), qui est cité plus de 80 fois dans $L P$ (noté p. xlii). Il a doté son ouvrage d'un apparat de notes incomparablement plus élaboré et plus fourni par rapport à After Life, où " the scholarly apparatus has been reduced to a minimum and as a rule indicates only the source of passages quoted in the text " (p. xii) : dans LP, en revanche, l'auteur indique systématiquement dans les notes de bas de page, ainsi que dans les notes complémentaires, toute la bibliographie pertinente sur chaque point particulier, restant à l'affût des nouveautés jusqu'aux dernières corrections d'épreuves. D'autre part, on a l'impression qu'il a mis un peu en sourdine la métempsychose et "The winning of immortality ", deux thèmes qui dans After Life avaient fait l'objet de sections bien identifiables. Il a même revu légèrement, mais de manière significative, certaines de ses propres positions, par exemple, en devenant plus prudent à l'égard du rôle joué par Posidonius (cf. p. xl-xli) ou en reconnaissant une influence grecque plus importante dans les cultes orientaux par rapport aux $R O$. Enfin et surtout, il a accordé à la philosophie un rôle prépondérant dans le processus étudié. Cela est palpable dans les longs développements consacrés à La critique philosophique (p. 145-179) - trop brièvement évoquée dans la "Historical introduction " d'After Life - et à la contribution du pythagorisme à l'élaboration des doctrines sur L'immortalité céleste (p. 181-228, où les Pythagoriciens figurent même dans le titre de la section I), mais avant tout dans le chapitre final consacré au Néoplatonisme (397-442). Ce chapitre, où C. "laisse éclater son émerveillement" (p. lix) devant l'élévation mystique du génial Plotin, qui l' "illuminait " à la fin de ses jours (n. 167), semble offrir non seulement le grand finale approprié à la thématique de l'ouvrage et son point d'orgue, mais véritablement la " clef de voûte de $[L P]$ et peut-être de toute l'œuvre de Cumont » $(\mathrm{p} . \mathrm{xl})^{17}$. On garde l'espoir que la réédition de $L P$ attirera de nouveau l'attention sur ces aspects novateurs et permettra la vraie redécouverte de l'ouvrage $^{18}$ : après tout, mieux vaut tard que jamais !

Évidemment, cela ne veut pas dire que la synthèse ambitieuse proposée par C. ne prête pas le flanc à la critique : A. Motte a consacré une section entière - incontestablement la plus substantielle de la deuxième partie, et celle qui interpelle le plus le lecteur moderne (p. cxxxiv-cxxxix) - à l'examen des Réserves et critiques formulées à l'égard

17 A. Motte note à juste titre (p. xcix, c et n. 490) la " caution » apportée par Émile Bréhier, éditeur-traducteur des Ennéades et grand spécialiste de Plotin, aux analyses philosophiques de Cumont dans son compte rendu de $L P$.

18 Par ricochet, on peut se demander en revanche quelle serait l'utilité - à part un intérêt purement historiographique, ou alors le souci louable d'exhaustivité... - de rééditer After Life (annoncé comme vol. 9 de la Bibliotheca Cumontiana, éd. J. Scheid et Ch. Frateantonio), à partir du moment où ces recherches encore embryonnaires furent grandement dépassées par $L P$ et le fait que la version anglaise du texte est loin d'avoir les qualités littéraires que l'on reconnaît à $L P$ - de plus, elle n'est pas directement de la main de Cumont (qui avait fait traduire son texte français). 
de $L P$, pas tellement dans les comptes rendus du livre ${ }^{19}$, mais surtout dans des publications plus récentes. Comme il est rappelé aussi dans les Conclusions (p. cxlii-cxliv), ces réticences viennent surtout de la part des historiens de la religion romaine (P. Veyne, J. Scheid), tandis que chez les historiens de la philosophie antique on constate une relative indifférence vis-à-vis de la problématique déployée dans $L P$ (p. cxlii, n. 490). En bref, sous trois points tiendraient les principales critiques qui lui sont adressées : (1) la surestimation de l'influence de l'Orient, à la fois concernant les cultes " orientaux " et l'origine iranienne de l'ascension céleste de l'âme ; (2) la surévaluation du symbolisme des sarcophages romains (critique adressée déjà à son Symbolisme funéraire); et (3) la fausse impression que l'on pourrait se faire des représentations gréco-romaines de la mort et de l'au-delà pendant les premiers siècles de l'Empire, du fait que C. privilégie les spéculations des philosophes et la thématique de l'immortalité au détriment des pratiques rituelles et des conceptions ordinaires, plus infra-mondaines, du commun des mortels, en ne laissant pas suffisamment apparaître la diversité du tableau. Ces points faibles de $L P$ méritent effectivement d'être repensés, revisités et réexaminés dans le détail par des recherches futures. Mais on doit souligner, à la défense de C., (1) que cette vision un peu déformante est due avant tout à la focalisation de sa recherche sur ce courant qui s'avère être minoritaire, et (2) que C. affirmait nettement et expressément que la croyance en l'immortalité céleste de l'âme ne fut pas dominante (p. 109-110 et suiv.). Néanmoins, les recherches de C. ont eu peut-être davantage un autre effet déformant dont on a beaucoup moins parlé - if at all -, à savoir la focalisation - par ailleurs légitime - de l'attention sur les périodes tardives, pendant lesquelles sont en gestation les mutations menant au christianisme. Le problème est que, ce faisant, on risque de " rater ", avec C., ce qui est une autre " révolution " incontestable : le " changement de paradigme " - pour le dire en des termes kuhniens - en matière de théories de l'âme et d'eschatologie, qui s'opère progressivement dans le monde grec pendant le $\mathrm{VI}^{\mathrm{e}}$ et $\mathrm{V}^{\mathrm{e}}$ siècle avant notre ère, avec la reconnaissance du caractère immortel de l'âme humaine, la métempsycose et les aspirations à la divinisation. Mais cela nous amènerait plutôt vers la Psyché de Rohde...

La doctrine étudiée par C. a fait aussi l'objet de recherches menées par les historiens de la Sternkunde (pour reprendre Fr. Boll) et du pythagorisme (deux secteurs malheureusement non explorés dans l'Introduction historiographique), et si l'on regarde de ce côté-là, on s'aperçoit vite que la référence à $L P$ y est incontournable ${ }^{20}$. On ne doit

19 Avec la notable exception du compte rendu détaillé de C. SCHNEIDER paru dans Gnomon 22 (1950), p. 201-204. Les points de critique apparaissant dans d'autres comptes rendus globalement positifs sont aussi dûment relevés par A. Motte.

20 Voir par exemple B. L. VAn der Waerden, Die Anfänge der Astronomie. Erwachende Wissenschaft II, Basel 1968, p. 204-252 et surtout W. BURKERT, Lore and science in ancient Pythagoreanism, Cambridge (Mass.) 1972, p. 357-368. Et pour le pythagorisme romain, l'ouvrage classique et toujours pas remplacé de L. Ferrero, Storia del pitagorismo nel mondo romano: dalle origini alla fine della repubblica, Torino 1955 (dont la réimpression 
pas oublier non plus que, presque immédiatement après leur parution, les recherches fondamentales de C. ont même donné à P. Boyancé et à M.P. Nilsson l'impulsion d'écrire deux articles importants sur la " religion astrale » et sur " immortalité astrale et mystique cosmique ", où ils rendent hommage au grand maître, tout en exprimant aussi des critiques à l'égard de certaines des positions avancées dans $L P^{21}$. Et que dire de la monumentale Révélation d'Hermès Trismégiste du père A.J. Festugière, publiée entre 1944 et 1954 (4 vol., 1750 p.) ? Non seulement son premier volume porte une dédicace à C., dont les travaux, abondamment cités dans les notes, auraient éveillé en Festugière un "ardent désir " (comme il le dit dans la préface $)^{22}$, mais les thèmes selon lesquels se décline cet ouvrage, qui est en réalité une somme sur la philosophie religieuse de l'époque hellénistique et impériale, apparaissent comme une série d'études inspirées par et complémentaires à la thématique de $L P$ (que Festugière connaissait déjà par les travaux antérieurs de C., en premier lieu After Life, le Symbolisme funéraire et le long article sur le « Mysticisme astral $\left.{ }^{23} »\right)$. Déjà les titres des trois premiers volumes en témoignent : L'astrologie et les sciences occultes, Le dieu cosmique, Les doctrines de l'âme; mais les développements consacrés au " mysticisme cosmique dans la littérature hermétique ", au Songe de Scipion, à l'" eschatologie " de l'âme incarnée ou à la " montée du voûs à l'Ogdoade" sont plus directement et plus intimement liés à $L P$.

Mais il est peut-être temps de voir maintenant comment a été reçue la réédition de LP par les contributeurs du volume intitulé Rome et ses religions. Walter Geerts, directeur de l'Academia Belgica de Rome, ouvre la discussion en rappelant utilement que l'achèvement de cet ouvrage-testament qu'est LP est allé de pair, en 1947, avec un autre acte testamentaire de grande valeur à la fois scientifique et symbolique, à savoir le

à Forlì en 2008 est malheureusement loin d'être satisfaisante ; elle a au moins l'avantage de comporter un bilan historiographique intéressant dû à A. BALBO, aux p. 9-16).

21 P. BOyAnCÉ, «La religion astrale de Platon à Cicéron ", Revue des études grecques 65 (1952), p. 312-350 (dédié explicitement "À la mémoire de Franz Cumont ") ; M.P. NiLsson, "Die astrale Unsterblichkeit und die kosmische Mystik », Numen 1 (1954), p. 106-119 [repris dans ID., Opuscula selecta, vol. 3, Lund 1960, p. 250-265]. Cf. aussi J. PÉPIN, "Cosmic piety ", dans A.H. Armstrong (éd.), Classical Mediterranean spirituality: Egyptian, Greek, Roman, New York 1986, p. 408-435.

22 Cf. H.D. SAfFrey, "Le Père André-Jean Festugière, O. P. (1898-1982) : portrait ", dans ID., Recherches sur le néoplatonisme après Plotin, Paris 1990, p. 297-305, aux p. 300-301. À la lumière de cette confidence, la phrase « une étincelle jaillit, et elle allume une flamme pour la vie entière », employée par Festugière dans un texte d'hommage à C. (cité p. cxi), acquiert indubitablement une forte résonance autobiographique.

23 Article présenté dans la préface du vol. 2 de la Révélation (p. xv) comme " un brillant mémoire » qui a "comme épuisé le sujet du mysticisme astral dans l'Antiquité ». 
don de la bibliothèque personnelle de C. - cette " fille illégitime " qu'il ne voulait pas voir " démembrée " - à l'Academia Belgica (voir Le don de la bibliothèque : une question de survie, p. 1-8). Par cet acte le grand savant offrait aux générations postérieures non seulement un véritable trésor d'érudition destiné à un usage public, mais aussi le meilleur moyen qui leur permettrait de suivre de près les traces de «son propre itinéraire scientifique tel que ses livres le reflètent » (p. 3).

Bruno Rochette se demande ensuite : Rééditer "Lux perpetua" : pour qui, pourquoi? (p. 9-20). Dans la première partie de sa contribution il propose un condensé substantiel de l'Introduction historiographique qu'il a rédigée avec André Motte, en soulignant combien la perspective de "l'œuvre achevée " doit avoir motivé l'investissement considérable du vieux C. dans la rédaction finale de $L P$. « Comme les bâtisseurs de cathédrales, [il] voulait sceller, au moyen d'une pierre angulaire, l'édifice scientifique qu'il avait passé sa vie à édifier ". Mais à travers cette œuvre "vivante et vivifiante " l'homme nous a laissé aussi " un témoignage authentique de son parcours intérieur ", illustrant ainsi parfaitement "l'union indispensable " entre science et vie, recherche scientifique et quête personnelle. Voilà déjà deux bonnes raisons qui justifient la décision de donner une deuxième vie à $L P$ telle qu'elle a été conçue et soigneusement préparée par son auteur - c'est-à-dire sans qu'elle soit mise à jour, revue et corrigée. Mais l'auteur en évoque aussi plusieurs autres : (1) $L P$ « reste toujours la seule synthèse sur les conceptions de l'au-delà " dans le monde gréco-romain ; (2) elle est " un témoin de son époque ", profondément enraciné " dans les préoccupations de son temps ", qui livre un "message d'espoir "; (3) plus qu'un exposé des résultats d'une enquête scientifique, elle est " une invitation à la réflexion sur un sujet qui touche tout être humain »; (4) le thème abordé dans cet ouvrage de la pleine maturité de C. constitue "l'un des fils conducteurs majeurs " de l'ensemble de sa "trajectoire scientifique "; (5) " ouvrage de grande ouverture et de grande culture ", $L P$ constitue, enfin, un modèle à suivre si l'on veut dépasser le morcellement et la compartimentation actuels du savoir.

Dans Les "Religions orientales " en mouvement : les ratures de Franz Cumont (p. 21-32), Sarah Rey présente et discute trois lettres inédites de C., trouvées dans les archives du Collège de France. Datant toutes de 1905 - l'époque où il préparait les conférences qui allaient donner naissance aux $R O$-, elles éclairent sa démarche intellectuelle et mettent le doigt sur ses hésitations terminologiques et conceptuelles. Trois des ratures qui y sont repérables s'avèrent particulièrement significatives : elles montrent un C. soucieux de remplacer (1) " culte " par " mystères " (à propos de Mithra) et (2) "paganisme gréco-latin » par "paganisme romain ", puis (3) de préciser que l'Orient fut toujours non pas vraiment le " centre » mais « la patrie la plus civilisée de l'empire romain ». S. Rey montre bien comment ces glissements révélateurs permettent d'appréhender (1) le caractère spécifique qui, aux yeux de C., distingue les cultes " orientaux » des autres cultes, à savoir " une intériorité cristallisée par les cérémonies des mystères, une émotion, une implication personnelle, une promesse individuelle 
qu'ignore la religion romaine "traditionnelle” "; (2) sa tendance à sous-estimer ${ }^{24}$ l'" étape grecque " dans sa "vision diffusionniste " des cultes d'origine orientale, au profit de leur réception romaine ; et (3) sa perception de l'Orient comme " berceau d'innovations cruciales pour le devenir de l'Occident ", d'un Orient qui, en dépit de son ambiguïté (notamment en matière de pratiques religieuses jugées superstitieuses), a joué en définitive un rôle déterminant dans le progrès moral et spirituel de l'humanité (y compris à travers la doctrine de l'immortalité astrale, selon la thèse développée dans $L P)$, et de ce fait n'est pas loin de se muer en un des principaux centres de gravité de l'approche cumontienne - et ce au détriment du christianisme qui se trouve finalement " contourné » dans l'analyse historique, à la fois comme comparandum et comme point d'aboutissement du processus.

Dans ce processus, C. pensait que les prêtres des cultes orientaux avaient joué un rôle capital en tant que prédicateurs et propagateurs de nouvelles doctrines et de croyances élevant moralement et spirituellement les âmes, en insistant en particulier sur la nécessité de purification de l'âme et en répandant "l'assurance qu'une immortalité bienheureuse serait la récompense de la piété " $(R O$, p. 59). C'est sur ces agents religieux que porte l'étude de Françoise van Haeperen, Des "médecins de l'âme ": les prêtres des "Religions orientales" selon Cumont (p. 49-62). L'auteure y examine la théorie extrêmement stimulante de C. selon laquelle, à la différence des collèges sacerdotaux romains traditionnels, les membres du clergé oriental auraient fonctionné aussi comme "directeurs de conscience ", prodiguant leurs conseils pour la réparation des fautes morales, ainsi que des " remèdes spirituels" pour les âmes souffrantes. Fr. van Haeperen semble prête à accepter la pertinence de cette théorie pour les prêtres d'Isis, les Mithriastes ou les prêtres syriens, cependant elle choisit à dessein le cas-limite des prêtres de Cybèle-Magna Mater pour soumettre la théorie généralisatrice de C. à ce qui ressemble à un crash test: en effet, dans ce cas, la tendance moralisante semble faire défaut ou être en dissonance avec une série de caractères " barbares " du mythe et du culte traditionnels (notamment l'auto-castration), ou alors elle n'apparaît que tardivement sous l'influence d'autres cultes (Mithra). C'est la perception cumontienne du personnel cultuel de Cybèle - très dépendante de l'image déformée qu'en donnaient les auteurs satiriques et les Pères de l'Église, et marquée par un « embarras larvé ... devant le statut des galles »- qui est approfondie dans la deuxième et plus longue partie de l'étude. On y apprend que l'examen attentif des sources invite à interpréter les rapports entre les différents acteurs du culte métroaque (archigalle, prêtre et prêtresse publics de la déesse, galles de Rome) non pas comme relevant d'une hiérarchie (selon « le modèle implicite des structures de l'Église catholique»), mais en termes de complémentarité de leurs fonctions respectives. Et sur le plan iconographique on est invité à reconnaître des

24 Sans pourtant l'ignorer complètement : voir l'importance accordée à l'étape alexandrine, ptolémaïque, dans ces lettres, et ce que Cumont écrira plus tard sur les mystères dans $L P$, p. 307. 
prêtres, voire des prêtresses de Cybèle dans les portraits aux traits efféminés supposés habituellement représenter des galli.

Dans LP le clergé des religions à mystères est aussi investi par C. d'une action unificatrice permettant de franchir le fossé qui sépare, dans le mouvement général de progression de l'histoire, les élites cultivées et rationnelles, moteurs de renouvellement et porteuses d'idées élevées, des strates populaires, attachées aux "vieilles croyances » superstitieuses. C'est cette action médiatrice et harmonisante qui rend possible une synthèse, et celle-ci est assurée également, selon C., par d'autres courants ou milieux : les mouvements mystiques du (néo)pythagorisme et du (néo)platonisme, ainsi que certains écrivains. Cela ressort très clairement de la contribution importante d'Annelies Lannoy, intitulée "Les masses vulgaires " et "les intelligences élevées " : les agents de la vie religieuse dans "Lux perpetua" et leur interaction (p. 63-81). L'auteure y souligne comment ces agents intermédiaires ont permis à $\mathrm{C}$. de nuancer la rigidité de " la dichotomie entre foules et intellectuels, tradition et évolution, émotivité et raison ", dont il se rendait compte qu'elle " n'est pas systématiquement applicable à la réalité romaine ", où des idées traditionnelles (voire des superstitions) pouvaient être présentes parmi les élites, tandis que des idées nouvelles venues des élites se glissaient parfois dans la pensée des masses.

À un autre niveau, et d'un autre point de vue, ces contradictions - qui sont toujours là pour nous rappeler que la réalité vivante des individus, des groupes, des sociétés a tendance à résister fortement à nos tentatives de " corsetage " et de schématisations simplificatrices, aussi fines soient-elles - s'expliquent peut-être par ce que Paul Veyne décrivait comme une "balkanisation des cerveaux » et Hendrik Versnel comme " inconsistance " ou " dissonance cognitive " interne. C'est la conclusion à laquelle arrive Robin Lane Fox dans Astrology and cognitive dissonance (p. 83-96), une contribution riche, bien documentée et attentive aux détails des sources, où l'auteur reprend le dossier traité par C. dans le chapitre VII de LP, L'astrologie et les morts prématurés. Lane Fox y met très bien en lumière comment le raisonnement déconfortant selon lequel " un destin inexorable ", " une fatalité omnipotente » règle tout «avec une rigueur mathématique » était atténué en réalité par ce que le grand spécialiste d'astrologie antique qu'était C. appelait respectivement " une doctrine aberrante » et " un illogisme flagrant ». À savoir (1) l'idée selon laquelle les enfants disparus " avant

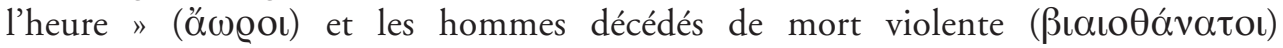
étaient condamnés à éprouver des souffrances " until their fixed span of time was fulfilled as astrologically determined at their birth " et (2) la croyance que "piety shown to ... the stars " - les agents mêmes du Destin ! - "would prove effective ", permettant ainsi aux âmes humaines d'échapper à la domination de la Nécessité.

Sur le plan historiographique, un moment fort des Actes est marqué par Michel Tardieu, excellent connaisseur de l'Altertumswissenschaft et de la Religionsgeschichtliche 
Schule dans leurs rapports avec les «théologiens modernes " en Allemagne ${ }^{25}$. Sa contribution sur La controverse de la "Mithrasliturgie " chez Cumont (p. 33-48) constitue un complément essentiel à l'Introduction historiographique de LP. Après un rappel de l'état actuel de la recherche au sujet de ce texte tiré du " grand papyrus magique de Paris " qu'Albrecht Dieterich avait interprété en 1903 comme une liturgie mithriaque, mais qui est en réalité une Recette d'immortalité ( $\alpha \pi \alpha \theta \alpha v \alpha \tau \iota \sigma \mu o ́ \varsigma$ ) produite probablement dans un milieu égyptien syncrétique à dominante pythagoricienne ${ }^{26}$, M. Tardieu examine dans le détail tous les aspects de cette controverse, à laquelle prirent part, outre C., plusieurs savants allemands de différents bords. L'auteur met notamment en lumière le sens provocateur, discrètement polémique, de la dédicace de Eine Mithrasliturgie à C. : convaincu d'avoir démontré que les étapes, décrites dans ce papyrus, "du parcours de l'initié dans les espaces sidéraux à la rencontre du dieu suprême étaient une sorte d'exégèse allégorique des étapes de l'initiation aux mystères romains de Mithra ", Dieterich semblait indiquer à C., alors maitre incontestable ès études mithriaques, qu'il "était passé à côté de la découverte à ne pas rater en histoire des religions ». C. y a répondu dans un compte rendu critique où il niait - à juste titre - l'interprétation mithriaque du document, mais Tardieu montre bien que ses arguments y sont tributaires de présupposés qui enlèvent beaucoup à leur valeur. Quant à la deuxième partie du livre de Dieterich, qui, à propos des principaux thèmes présents dans le papyrus, offre " une morphologie complète des représentations religieuses » (Fr. Boll), et que C. lui-même jugea " très remarquable ", elle pourrait être véritablement, selon M. Tardieu, "le germe " d'où naîtra $L P$ - notamment les chapitres finaux consacrés à la "Wiedergeburt» et à la "Himmelfahrt der Seele zu Gott». Ainsi LP «peut apparaître comme une sorte d'anti-Mithrasliturgie, ou plutôt comme la refonte des thèmes qui font l'objet des deux ouvrages de Dieterich sur l'immortalité céleste et le voyage dans l'au-delà, Nekyia et Mithrasliturgie ".

À la fin de son étude, Tardieu remarque que, contrairement à Dieterich, C. "place au point de départ [...] la critique philosophique de la religion et la perception des mutations de la pensée religieuse par la philosophie ». Danny Praet, actuellement plongé dans la préparation du premier volume des Kleine Schriften cumontiennes ${ }^{27}$, portant sur L'histoire de la philosophie (surtout le pythagorisme et la tradition platonicienne), illustre parfaitement cela en étudiant Les liens entre philosophie et religion dans

25 Voir la très substantielle préface qu'il a rédigée pour la version française d'A. vON HaRnack, Mission et expansion du christianisme aux trois premiers siècles, trad. J. Hoffmann, Paris 2004 (référence " cachée " dans Actes, p. 40, n. 27 ; cf. p. 35, n. 10 : la référence à HARNACK 2004 ne correspond à rien dans la bibliographie finale de l'article).

26 Voir M. ZAGO (éd.), Anonimo. La ricetta di immortalità : testo greco a fronte, Milano 2010 ; cf. aussi EAD., "Un portrait de Pythagore dans la Liturgie de Mithra ", Zeitschrift für Papyrologie und Epigraphik 177 (2011), p. 53-57.

27 Sur le projet de réédition des scripta minora de Cumont dans leur ensemble, voir Actes, p. 106-107 et http://www.cumont.ugent.be/en. 
quelques Scripta minora de Franz Cumont (p. 97-110). Son étude vient compléter les pages de l'Introduction historiographique de $L P$ (p. xlii-xlix) consacrées aux articles dans lesquels $\mathrm{C}$. avait déjà commencé à aborder certains des thèmes - souvent à résonance philosophique - qui ont fini par être intégrés dans la summa que constitue $L P$. D. Praet veut surtout saisir la «tension dialectique » qui, selon C., traverse l'histoire de la pensée antique, " entre une philosophie rationaliste, matérialiste, qui détruit les traditions religieuses » - exposée principalement dans le chap. II de $L P$ (p. 145-178), portant sur les philosophies hellénistiques - et « une philosophie novatrice, plutôt idéaliste et propagatrice d'un mysticisme réfléchi ", qui correspond au pythagorisme renaissant qui a ouvert la voie au néoplatonisme (voir surtout le chap. VIII, p. 397-442). Il montre bien, en comparant le mémoire de jeunesse (1887) de C. sur Alexandre d'Abonotique, le «faux prophète " de Lucien, et sa (quasi) retractatio sur le sujet 35 ans plus tard (1922), que cette tension habitait en réalité C. lui-même. C. semble en effet avoir évolué au fil du temps, passant d'une attitude anticléricale de type voltairien, "plutôt hostile envers beaucoup de manifestations du phénomène religieux ", en tout cas envers celles qui lui paraissaient superstitieuses et qu'il associait à l'obscurantisme, à une attitude plus empathique à l'égard du religieux (explicable en partie par « le désillusionnement envers le rationalisme moderne provoqué par la Première Guerre mondiale "); surtout à partir du moment où, grâce à l'influence de courants comme le néo-pythagorisme, la religiosité ne se limitait plus à des "pratiques purement rituelles ou magiques ", mais devenait " une religion d'initiés où la science $(\gamma \nu \omega \tilde{\omega} \sigma \mathrm{s})$ était à la fois récompense de la piété et la fin à laquelle l'homme devait aspirer " et où " pour l'obtention du salut » l'on exigeait "de rigoureuses conditions morales " (p. 100, citant C.).

Cette évolution personnelle de C. explique en grande partie le «sentiment d'altérité " qu'il semble avoir éprouvé pour la philosophie hellénistique, "qui représente pour lui, en règle générale, la victoire d'une raison ennemie des croyances traditionnelles ", par opposition au pythagorisme (et ses avatars plus tardifs) et à la tradition platonicienne dans son ensemble (Platon, l'ancienne Académie, le moyen platonisme et le néoplatonisme), où $\mathrm{C}$. retrouve "l'orientation philosophique qui lui est chère ". C'est ce que montre très bien Carlos Lévy dans Franz Cumont et les pensées de l'immanence (p. 111-124), où il examine la façon dont C. appréhendait le scepticisme, l'épicurisme (surtout Lucrèce et Philodème de Gadara) et le stoïcisme (surtout Panétius et Posidonius). Sa conclusion est que, du point de vue de l'histoire de la philosophie, on doit faire attention aux interprétations avancées par C., car elles ne sont pas toujours exemptes de généralisations parfois schématiques, de focalisations inattendues, d'a priori ou de jugements de valeur, et même d'inexactitudes ou d'erreurs - aspects mis en relief par C. Lévy avec le savoir, la justesse et la perspicacité qui sont les siennes. Mais j'aimerais attirer ici l'attention sur un moment de générosité particulière de sa part à l'égard de C., lorsqu'il écrit : "À la différence du courant analytique actuel, Cumont intègre toujours la philosophie au grand tout de l'histoire, sans négliger aucun de ses aspects (événements, mentalité, religion, grands courants artistiques et intellectuels) ", en revendiquant pour elle une ambition holistique qui suppose « une prise en charge de 
l'homme total, depuis les pulsions les plus obscures du subconscient jusqu'aux formes les plus hautes de la rationalité ». C'est effectivement une perspective «stimulante et rafraîchissante " en vertu de laquelle le " beau risque " qu'a couru C. mériterait peutêtre d'être couru de nouveau par la recherche contemporaine - mais cette fois-ci en connaissance de cause et en profitant de tous les développements de la science intervenus entre temps, depuis la "psychologie religieuse ", l'anthropologie et l'histoire des mentalités, jusqu'à l'histoire des idées et l'approche de la philosophie comme mode de vie, sans oublier la contribution encore plus récente des sciences cognitives.

Les aspects encore stimulants de l'œuvre de C., qui justifient pleinement sa réédition dans une optique résolument historiographique, sont soulignés également dans la contribution qui clôt l'ensemble, due à celle qui a tant œuvré pour les études cumontiennes : Corinne Bonnet ("Lux perpetua " : un testament spirituel ?. p. 125-141). Mais avant d'en arriver là, elle offre, "de l'intérieur " en quelque sorte, étant donné sa longue familiarité avec l'œuvre et la personnalité de son éminent compatriote, quelques considérations globales sur le parcours scientifique mais aussi existentiel de C. : d'une part, en revenant sur l'idée que $L P$ est «l'aboutissement naturel d'un long cheminement scientifique cohérent » et en s'attardant sur certains points illustrant ce "va-et-vient entre passé et présent qui nourrit le livre à plusieurs niveaux "; d'autre part, en tentant de saisir les convictions intimes de C. ${ }^{28}$, " ce qui s'agitait en son "for intérieur", dans cet "asile inviolable" de sa pensée " - en restant lucide sur les limites d'une telle démarche, mais "prêchant " résolument pour " une lecture déconfessionnalisée » de C. et indiquant in fine " quelque piste nouvelle que les exégètes ont comme miraculeusement laissée en friche ». Du point de vue de l'histoire des religions, ce que l'auteure met bien en évidence, c'est le statut singulier qu'accorde C. à la religion : dotée de sa propre dynamique sui generis, elle transcende selon lui les phénomènes purement matériels, étant avant tout affaire de spiritualité, de croyance, de conviction et d'options éthiques fondamentales à vocation universelle ; spiritualité et morale en sont les fondements. C'est dans cette perspective qu'il faut situer et comprendre les recherches de C. sur les conceptions funéraires et eschatologiques des anciens, dont les prémices remontent - et c'est un point crucial qui n'avait pas été remarqué auparavant, je crois - à l'époque où les images du culte de Mithra (son "premier amour ", auquel il se consacra entre 1894 et 1899) «l'orientent vers une symbolique astrale, et même un mysticisme astral ", Mithra " charri[ant] ", en " héritier du dualisme zoroastrien ", "des idées nouvelles sur le destin des âmes initiées qui voyagent vers des sphères supérieures ». Quant aux convictions personnelles de C. et au message qu'il a voulu laisser à travers $L P$, son testament spirituel, il faut surtout se défaire, selon C. Bonnet, du Cumont christianisé qui nous est restitué dans la préface de $L P$ rédigée par $\mathrm{L}$. Canet et ne pas se laisser égarer par l'idée que l'étude des religions païennes, dans ce qu'elles ont

28 Sans doute dans l'idée que $L P$ « exprime certainement [l] es convictions les plus profondes » de C. (ainsi J. ScHEID, Actes, p. vii). 
produit de plus profond et spirituel, ne serait au bout du compte qu' " une propédeutique, une praeparatio evangelica". Pour avoir quelque chance de saisir le vrai C., dit C. Bonnet, c'est vers des textes de lui qu'il faut se tourner. Mais lesquels ? Ni catholique ni franc-maçon ${ }^{29}$, C. aurait peut-être investi dans l'élévation philosophique des auteurs néoplatoniciens, surtout celle de Plotin, à l'égard de qui il exprimait à chaque occasion une admiration inconditionnelle, en raison de la combinaison idéale de rationalisme grec et de spiritualité mystique à laquelle il semblait être parvenu ?

C. Bonnet pense que la clé nous est donnée plutôt dans la belle Introduction que C. a rédigée pour $L P$ (p. 31-42). En effet, si l'on relit son évocation vibrante des mystères du cosmos tel que l'avait découvert l'astronomie moderne et sa méditation sur les changements de paradigme et les profonds bouleversements de perspective qui en découlent pour l'homme, à la lumière d'un acquis incontestable de l'étude menée dans $L P$, à savoir que "l'eschatologie des Anciens [...] épouse les contours de leur cosmologie ", on s'apercevra que la perspective de C. " est bien davantage cosmique que chrétienne ». On ne peut pas s'empêcher alors de se demander quel type de religiosité et quelle perspective eschatologique seraient selon lui les mieux adaptées à la nouvelle cosmologie du $x^{\mathrm{e}}$ siècle, autrement dit dans quel sens il interpréterait lui-même aujourd'hui l'expression lux perpetua...

\section{Addenda bibliographiques ${ }^{30}$ (par ordre alphabétique des thèmes)}

- Âme et éther : A. MinAI, "Soul's aitherial abode according to the Poteidaia epitaph and the Presocratic philosophers ", Numen 57 (2010), p. 553-582.

- Âme et souffle : E. BARRA-SALzÉDO, En soufflant la grâce (Eschyle, Agamemnon, v. 1206) : âmes, souffles et humeurs en Grèce ancienne, Grenoble 2007. * A. Bernabé, J. MendozA, "Pythagorean cosmogony and Vedic cosmogony (RV10.129) : analogies and differences", Phronesis 58 (2013), p. 32-51.

- Âme (théories philosophiques) : D. Frede, B. ReIs (éd.), Body and soul in ancient philosophy, Berlin / New York 2009.

- Apocalyptique juive : S. C. Mimouni, A. Sérandour (éd.), La littérature apocalyptique : entre prophétisme, messianisme et millénarisme?, Revue des études juives 169.1-2 (2010).

29 Voir C. Bonnet, «Un Cumont peut en cacher un autre : à propos de l'appartenance de Franz Cumont à la franc-maçonnerie ", Anabases 8 (2008), p. 197-203.

30 À ajouter à ceux, déjà très importants, proposés dans les notes de bas de page et l'Appendice bibliographique (p. cxliv-cxlviii) de l'Introduction historiographique de LP. Il aurait été souhaitable que le classement thématique à l'intérieur de cet appendice ait été plus détaillé, en sorte que le lecteur puisse en dégager plus aisément les références pertinentes pour chaque sujet particulier. 
- Archéologie funéraire : J. BAZANT, «Entre la croyance et l'expérience : le mort sur les lécythes à fond blanc ", Bulletin de correspondance hellénique - Supplément 14 (1983), p. 37-44. * Chr. Goudineau (éd.), Rites funéraires à Lugdunum, Paris 2009. * J. SCHEID (éd.), Pour une archéologie du rite : nouvelles perspectives de l'archéologie funéraire, Rome 2008. * VAN ANDRINGA et al., Mourir à Pompéi. Fouille d'un quartier funéraire de la nécropole romaine de Porta Nocera (2003-2007), Rome 2013.

- Astrologie : T. S. Barton, Ancient astrology, London / New York 1994. * EAD., Power and knowledge : astrology, physiognomics and medicine under the Roman Empire, Ann Arbor 1994. * R. GoRDON, "Cosmology, astrology, and magic : discourse, schemes, power, and literacy ", dans L. Bricault et C. Bonnet (éd.), Panthée : religious transformations in the Graeco-Roman empire, Leiden - Boston 2013, p. 85-111. * S.B. Noegel, J. Th. Walker, B. M. Wheeler (éd.), Prayer, magic, and the stars in the ancient and late antique world, University Park (Pennsylvania) 2003.

- Astrologie et Mithra : R. BECK, Planetary gods and planetary orders in the mysteries of Mithras, Leiden 1988. * ID., Beck on Mithraism : collected works with new essays, Aldershot 2004. * ID., The religion of the Mithras cult in the Roman Empire : mysteries of the unconquered sun, Oxford 2006.

- Banquet funéraire : J.-M. Dentzer, Le motif du banquet couché dans le ProcheOrient et le monde grec antique du VII au IV siècle avant J.-C., Rome 1982.

- Catabases: W. BurKerT, "Parmenides' proem and Pythagoras descent ", dans V. Adluri (éd.), Philosophy and salvation in Greek religion, Berlin 2013, p. 85-116 (original allemand 1969). * M.L. Gemeli Marciano (et al.), Parmenide : suoni, immagini, esperienza (éd. L. Rossetti, M. Pulpito), Sankt Augustin 2013. * P. Kingsley, Dans les antres de la sagesse : études parménidiennes, trad. H.D. Saffrey, Paris 2007 (original anglais 1999). * M.A. Santamaría Álvarez, « La catábasis de Pitágoras », dans T. Calvo Martínez, Fr. Casadesús (éd.), Actas del Primer Congreso Internacional de la Sociedad Ibérica de Filosofia Griega, Palma de Mallorca, 24-26 de abril de 2008, p. 565-573 (à paraitre). * Y. Ustinova, Caves and the ancient Greek mind: descending underground in the search for ultimate truth, Oxford 2009.

- Catastérismes : J. PÀmIAS i MASSANA (introd., éd. crit., trad. \& n.), Eratòstenes de Cirene. Catasterismes, Barcelona 2004.

- Charon : S. T. STEVEns, "Charon's obol, and other coins in ancient funerary practice ", Phoenix 45 (1991), p. 215-229.

- Christianisation : M.-F. BASLEZ, Comment notre monde est devenu chrétien, Paris 2008. * J. N. BREMMER, The rise of Christianity through the eyes of Gibbon, Harnack and Rodney Stark, Groningen 2010. * D. PRAET, "Explaining the Christianisation of the Roman Empire : older theories and recent developments ", Sacris Erudiri. Jaarboek voor Godsdienstwetenschappen 33 (1992-1993), p. 5-119. * P. VEYNE, Quand notre monde est devenu chrétien (312-394), Paris 2007.

- Cosmologie, sotériologie, eschatologie et rituel funéraire (papyrus de Derveni, Empédocle, ...) : G. Betegh, "Papyrus on the pyre : the Derveni papyrus and its archaeological context ", Acta antiqua Academiae scientiarum Hungaricae 42 (2002), 
p. 51-66. * ID., The Derveni papyrus : cosmology, theology and interpretation, Cambridge 2004. * ID., "Eschatology and cosmology : models and problems ", dans M. M. Sassi (éd.), La costruzione del discorso filosofico nell'età dei Presocratici / The construction of philosophical discourse in the age of the Presocratics, Pisa 2006, p. 27-50. * A. LAKs, "Between religion and philosophy : the function of allegory in the Derveni papyrus", Phronesis 42 (1997), p. 121-142. * G.W. Most, "The fire next time : cosmology, allegoresis, and salvation in the Derveni papyrus ", Journal of Hellenic Studies 117 (1997), p. 117-135. * D. OBBINK, "Cosmology as initiation vs. the critique of Orphic mysteries ", dans A. Laks, G.W. Most (éd.), Studies on the Derveni papyrus, Oxford 1997, p. 39-54. * I. Papadopoulou, L. Muellner (éd.), Proceedings of the Derveni papyrus Conference (Center for Hellenic Studies, July 7-9 2008), Classics@: An online Journal 5 (2011) : http://chs.harvard.edu/wa/pageR?tn=ArticleWrapper\&bdc=12\&m $\mathrm{n}=2653$. * V. Piano, "Le papyrus de Derveni et son contexte de découverte : parole écrite et rituels funéraires dans la Macédoine grecque antique ", Revue de l'histoire des religions 230.2 (2013), p. 233-252. * Rhizai. A Journal for Ancient Philosophy and Science 4.1 (2007), p. 1-162 (numéro spécial sur le papyrus de Dervéni). * R. SEAFORD, "Immortality, salvation, and the elements ", Harvard Studies in Classical Philology 90 (1986), p. 1-26. * P. SKarsoul, « Des papyri grecs en contexte funéraire : l'exemple de l'"Empédocle de Strasbourg" ", Mètis (N.S.) 8 (2010), p. 167-187.

- Crémation : J. Musgrave, "Dust and damn'd oblivion : a study of cremation in ancient Greece ", The Annual of the British School at Athens 84 (1990), p. 271-299.

- Dionysos (mystères) : A.-Fr. JAссотTет, Choisir Dionysos : les associations dionysiaques ou la face cachée du dionysisme, 2 vol., Zürich 2003.

- Divinisation / apothéose : K. Buraselis et al., art. "Heroisierung und Apotheose ", ThesCRA 2 (2004), p. 125-214. * C. LÉvy (éd.), Devenir dieux : désir de puissance et rêve d'éternité chez les Anciens, Paris 2010. * M. J. Vermaseren, Liber in deum : l'apoteosi di un iniziato dionisiaco, Leiden 1976.

- Égypte : J. Assmann, Images et rites de la mort dans l'Égypte ancienne : l'apport des liturgies funéraires, Paris 2000. * ID., Mort et au-delà dans l'Égypte ancienne, Paris 2003 (original allemand 2001).

- Éleusis : F. Graf, Eleusis und die orphische Dichtung Athens in vorhellenistischer Zeit, Berlin 1974.

- Épigrammes funéraires : A. LE BRIS, La mort et les conceptions de l'au-delà en Grèce ancienne à travers les épigrammes funéraires : étude d'épigrammes d'Asie mineure de l'époque hellénistique et romaine, Paris 2001. * É. WOLFF, La poésie funéraire épigraphique à Rome, Rennes 2000.

- Épinomis : Fr. Alesse, Fr. Ferrari (éd.), Epinomide. Studi sull'opera e la sua ricezione, Napoli 2012.

- Er (mythe d') : Fr. Calabi, "Il mito di Er : fontes ", dans M. Vegetti (éd.), Platone. La Repubblica : traduzione e commento, vol. 7 : Libro X, Napoli 2007, p. 277-310. 
- Faute ancestrale : R. GaGné, Ancestral fault in ancient Greece, Cambridge 2013.

- Hadès, Enfers et au-delà (représentations de) : A. Alvino, "L'invisibilità di Ades ", Studi storico-religiosi 5 (1981), p. 45-51. * A. BAllabriga, Le Soleil et le Tartare: l'image mythique du monde en Grèce archä̈que, Paris 1986. * A. CHaniotis, "Das Jenseits - eine Gegenwelt ?", dans T. Hölscher (éd.), Gegenwelten zu den Kulturen der Griechen und der Römer in der Antike, Munich-Leipzig 2000, p. 159-181. * C. Cousin, La représentation de l'espace et du paysage des enfers en Grèce jusqu'au IV'eme siècle avant J.-C. : étude littéraire et iconographique, microforme, Lille 1996. * Jenseitsvorstellungen in Antike und Christentum: Gedenkschrift für Alfred Stuiber, Münster 1982. * E. KEULS, The water carriers in Hades : a study of catharsis through toil in classical Antiquity, Amsterdam 1974. * P. KInGSLEY, Empédocle et la tradition pythagoricienne : philosophie ancienne, mystère et magie, trad. G. Lacaze, Paris 2010 (original anglais 1995). M. LABAHn, M. LANG (éd.), Lebendige Hoffnung - ewiger Tod ? Jenseitsvorstellungen im Hellenismus, Judentum und Christentum, Leipzig 2007. * Th. ReYser, Discours et représentations de l'au-delà dans le monde grec, Thèse de Doctorat, Université Paris-Est 2011 (dir. M.-Fr. Baslez).

- Héraclide le Pontique : I. Kupreeva, "Heraclides' On Soul (?) and its ancient readers ", dans W.W. Fortenbaugh, E. Pender (éd.), Heraclides of Pontus : discussion, New Brunswick (N.J.) London 2009, p. 93-138. * H.A.T. ReICHE, « Heraclides' three soul-gates : Plato revised ", Transactions of the American Philological Association 123 (1993), p. 161-180.

- Immortalité : A. H. Armstrong, Expectations of immortality in late Antiquity (The Aquinas lecture), Milwaukee 1987. * Fr. Casadesús Bordoy, "On the origin of the Orphic-Pythagorean notion of the immortality of the soul ", dans G. Cornelli, R. McKirahan, C. Macris (éd.), On Pythagoreanism, Berlin 2013, p. 153-176. * E. Ehnmark, "Some remarks on the idea of immortality in Greek religion ", Eranos 46 (1948), p. 1-21. * Religions et histoire $\mathrm{n}^{\circ}$ 52, Sept./Oct. 2013, L'immortalité : croyances et pratiques dans les religions du monde, de la préhistoire à nos jours.

- Immortalité - Zalmoxis : D. DANA, "Zalmoxis et la quête de l'immortalité : pour la révision de quelques théories récentes ", Les études classiques 75 (2007), p. $93-110$.

- Iran ancien et Magi (influence sur le monde grec) : S.M.R. DARBANDI, A. Zournatzi (éd.), Ancient Greece and ancient Iran : cross-cultural encounters. Proceedings of the First International Conference (Athens, 11-13 November 2006), Athens 2008. * Fr. Ferrari, "Rites without frontiers : Magi and mystae in the Derveni papyrus ", Zeitschrift für Papyrologie und Epigraphik 179 (2011), p. 75-82. * Ph. S. Horky, "Persian cosmos and Greek philosophy: Plato's associates and the Zoroastrian Magoi", Oxford Studies in Ancient Philosophy 37 (2009), p. 47-103. * P. KingSLEY, "The Greek origin of the sixth-century dating of Zoroaster ", Bulletin of the School of Oriental and African Studies 53 (1990), p. 245-265. * Id., "Greeks, shamans and Magi ", Studia Iranica 23 (1994), p. 187-198. * ID., " Meetings with Magi : Iranian 
themes among the Greeks, from Xanthus of Lydia to Plato's Academy ", Journal of the Royal Asiatic Society (Ser. 3) 5 (1995), p. 173-209. * A. PANAINO, "Considerazioni sulla trasmissione delle scienze e delle cosiddette "pseudo-scienze" tra Grecia e Iran ", dans C.G. Cereti, B. Melasecchi, F. Vajifdar (éd.), Varia Iranica, Roma 2004, p. 213-237. * ID., "L'aldilà zoroastriano e quello dantesco. Appunti per una riflessione comparativa e tipologica su forme e motivi ricorrenti nei viaggi ultraterreni ", dans A. Cottignoli, D. Domini, G. Gruppioni (éd.), Dante e la fabbrica della Commedia, Ravenna 2008, p. 171-187. * ID., "I Magi in Occidente ", dans G.M. Cazzaniga (éd.), Storia d'Italia. Annali, vol. 25. Esoterismo, Torino 2010, p. 49-76. * ID., "Apocalittica, escatologia e sciamanismo nell'opera iranologica di Ph. Gignoux. Con una nota sulla "visione" del mago Kirder ", dans R. Gyselen, Chr. Jullien (éd.), "Maître pour l'éternité ». Florilège offert à Philippe Gignoux pour son $80^{\circ}$ anniversaire, Paris 2011, p. 205-243. * J.R. RusselL, "The Magi in the Derveni papyrus ", Name-ye Iran-e Bastan 1.1 (2008), p. 49-59.

- Judaïsme : A. J. Avery-Peck, J. Neusner (éd.), Judaism in Late Antiquity, vol. 4. Death, life-after-death, resurrection and the world-to-come in the Judaisms of Antiquity, Leiden/Boston/Köln 2000. * J. CosTA, L'au-delà et la résurrection dans la littérature rabbinique ancienne, Paris-Louvain 2004. * E. PUECH, La croyance des Esséniens en la vie future : immortalité, résurrection, vie éternelle? Histoire d'une croyance dans le judaïme ancien, Paris 1993.

- Jugement infernal : J. AnNAs, "Plato's myths of judgement ", Phronesis 39 (1994), p. 119-143.

- Lamelles d'or : R.G. Edmonds III (éd.), The "Orphic " gold tablets and Greek religion : further along the path, Cambridge 2011. * C. Russo, "Dalla morte alla "vera vita" : revisione autoptica della lamina di Hipponion ", Epigraphica 58 (1996), p. 35-59. * M. Tortorelli Ghidini, Figli della Terra e del Cielo stellato. Testi orfici con traduzione e commento, Napoli 2006. * Y. TzIFopoulos, "Paradise " earned: the Bacchic-Orphic gold lamellae of Crete, Cambridge (Mass.) 2008.

- Lune : S. LunAïs, Recherches sur la lune. I. Les auteurs latins, de la fin des guerres puniques à la fin du règne des Antonins, Leiden 1979. * J.-Cl. РiCOT, "Empédocle pouvait-il faire de la lune le séjour des Bienheureux ?", Organon (Warszawa), 37 (40), 2008, p. 9-38. * Cl. PréAux, La lune dans la pensée grecque, Bruxelles 1973.

- "Moralisation " (processus de) : A. Bernabé, "L'âme après la mort : modèles orphiques et transposition platonicienne ", Études Platoniciennes 4 (2007) [Les puissances de l'âme selon Platon], p. 25-44. * A. Chaniotis, "Greek ritual purity : from automatisms to moral distinctions ", dans P. Rösch, U. Simon (éd.), How purity is made, Wiesbaden 2012, p. 123-139.

- Mort - Bibliographie : N. CRINITI, "Mors antiqua: bibliografia sulla morte e il morire a Roma ", Ager Veleias 5.10 (2010), p. 1-30 : http:/www.veleia.it/download/ allegati/fn000298.pdf. * ID., "Mors moderna : bibliografia orientativa sulla morte e il morire nel mondo occidentale ", Ager Veleias 6.01 (2011), p. 1-24 : http://www.veleia. it/download/allegati/fn000414.pdf. * ID., " Memoria mortuorum nel mediterraneo 
antico ", Ager Veleias 6.04 (2011), p. 1-35 : http://www.veleia.it/download/allegati/ fn000416.pdf.

- Mort en Grèce antique (représentations et attitudes) : B. C. Dietrich, Death, fate, and the gods : the development of a religious idea in Greek popular belief and in Homer, London 1965. * I. MorrIs, "Attitudes toward death in archaic Greece ", Classical Antiquity 8 (1989), p. 296-320. * N.J. RiCHARDSON, " Early Greek views about life after death ", dans P.E. Easterling, J.V. Muir (éd.), Greek religion and society, Cambridge 1985, p. 50-66. * A. SAmellas, Death in the Eastern Mediterranean (50-600 A.D.) : the Christianization of the East. An interpretation, Tübingen 2002.

- Morts des philosophes dans la tradition biographique : Chl. Balla, V. Kotzia,

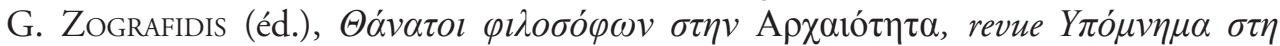

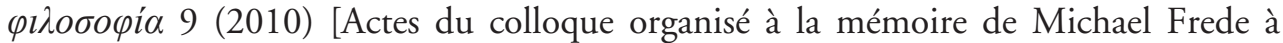

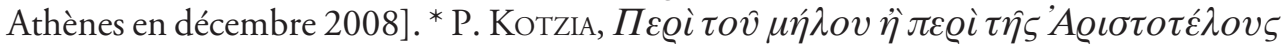
$\tau \varepsilon \lambda \varepsilon v \tau \hat{\jmath}$ (Liber de pomo), Thessaloniki 2007.

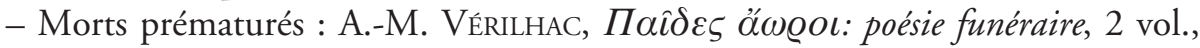
Athènes 1982.

- Mutations religieuses : L. Bricault, C. Bonnet (éd.), Panthée : religious transformations in the Graeco-Roman empire, Leiden/Boston, 2013. * E. R. DodDs, Païens et chrétiens dans un âge d'angoisse : aspects de l'expérience religieuse de Marc-Aurèle à Constantin, trad. H.D. Saffrey, Paris ${ }^{2} 2010$ [ [1979] (original anglais 1965).

- Mystères (eschatologie) : G. Sfameni Gasparro, "Après Lux perpetua de Franz Cumont : quelle eschatologie dans les "cultes orientaux" à mystères? ", dans L. Bricault, C. Bonnet (éd.), Panthée : religious transformations in the Graeco-Roman empire, Leiden/ Boston, 2013, p. 145-167.

- Mystique dans l'Antiquité : S. C. Mimouni, M. Scopello, A. Sérandour, C. MACRIs (éd.), Mystique théorétique et mystique théurgique dans l'Antiquité gréco-romaine : paganismes, judaïsmes, christianismes, Paris (à paraître en 2014 dans la coll. " Mystica " des éd. Honoré Champion).

- Néoplatonisme : S. R. Ph. GerTz, Death and Immortality in Late Neoplatonism: Studies on the Ancient Commentaries on Plato's "Phaedo ", Leiden 2011.

- Néopythagorisme : J.-J. Flinterman, «From Nigidius Figulus to Alexander of Abonouteichos: Pythagoreans in Rome and Asia Minor around the turn of the common era ", dans C. A. Huffman (éd.), A history of Pythagoreanism, Cambridge (à paraître en 2014 à Cambridge Universiy Press). * J. A. NorTH, "Sappho underground ", dans B. Dignas et R. R. R. Smith (éd.), Historical and Religious Memory in the Ancient World, Oxford 2012, p. 37-67 (à propos de ladite "basilique pythagoricienne " de la Porta Maggiore à Rome). * M. Schofield (éd.), Aristotle, Plato and Pythagoreanism in the first century BC: New directions for philosophy, Cambridge 2013. * P. VESPERINI, La philosophia et ses pratiques d'Ennius à Cicéron. Rome 2012.

- Numénius : P. P. Fuentes GonzÁlez, "Nouménios (Numénius) d’Apamée ", dans R. Goulet (éd.), Dictionnaire des philosophes antiques, vol. IV, Paris 2005, p. 724-740. 
- Oracles chaldaïques: H. Seng, M. TARdieu (éd.), Die Chaldaeischen Orakel: Kontext - Interpretation - Rezeption, Heidelberg 2010.

- Orient (influence sur la Grèce) : W. Burkert, Babylon, Memphis, Persepolis : Eastern contexts of Greek culture, Cambridge (Mass.) / London 2004. * W. BURKERT, "Prehistory of Presocratic philosophy in an Orientalizing context", dans P. Curd, D. Graham (éd.), The Oxford Handbook of Presocratic Philosophy, Oxford 2008, p. 55-86. * Chr. RIEDWEg (éd.), Grecia Maggiore : intrecci culturali con l'Asia nel periodo arcaico. . Atti del simposio in occasione del 75. anniversario di Walter Burkert = Graecia Maior: Kulturaustausch mit Asien in der archaischen Periode. Akten des Symposions aus Anlass des 75. Geburtstages von Walter Burkert (Istituto Svizzero di Roma, 2.2.2006), Basel 2009.

- Orphica : A. Bernabé (éd.), Poetae epici graeci. Pars II. Fasc. 1-2. Orphicorum et orphicis similium testimonia et fragmenta, Munich / Leipzig 2004-2005. Fasc. 3. Musaeus, Linus, Epimenides, Papyrus Derveni, indices, Berlin 2007. * F. Graf, « Text and ritual : the corpus eschatologicum of the Orphics", dans G. Cerri (éd.), La letteratura pseudepigrafa nella cultura greca e romana. Atti di un incontro di studi, Napoli 15-17 gennaio 1998 = Annali dell'Istituto Universitario Orientale di Napoli. Dipartimento di Studi del Mondo Classico e del Mediterraneo Antico 22 (2000), p. 59-77. * M. Herrero De Jáuregui, «Las fuentes de Clem. Alex. Protr. 2.12-22 : un tratado sobre los misterios y una teogonia orfica ", Emerita 75.1 (2007), p. 1-32. * ID., " Orphic ideas of immortality : traditional Greek images and a new eschatological thought ", dans M. Labahn, M. Lang (éd.), Lebendige Hoffnung - ewiger Tod ? Jenseitsvorstellungen im Hellenismus, Judentum und Christentum, Leipzig 2007, p. 247-273. * M. MarINČIC, " Der "orphische" Bologna-Papyrus (Pap. Bon. 4), die Unterweltsbeschreibung im Culex und die lukrezische Allegorie des Hades ", Zeitschrift für Papyrologie und Epigraphik 122 (1998),

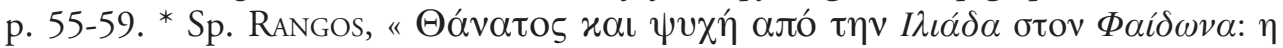

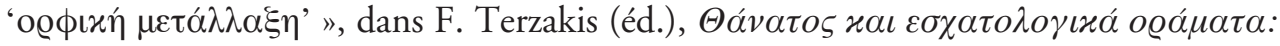

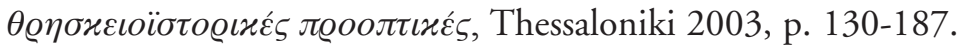

- Perséphone : G. ZunTz, Persephone : three essays on religion and thought in Magna Graecia, Oxford 1971.

- Philosophie et religion : P. AthanAssiadi et C. Macris, « La philosophisation du religieux ", dans L. Bricault, C. Bonnet (éd.), Panthée : religious transformations in the Graeco-Roman empire, Leiden/Boston 2013, p. 41-83.

- Pindare - eschatologie : L. EDMUNDS, "A hermeneutic commentary on the eschatological passage in Pindar Olympian 2 (57-83) ", dans Chr. Walde, U. Dill (éd.), Antike Mythen: Medien, Transforamtionen und Konstruktionen, Berlin 2009, p. 662-677. * H. LLoyd-JoneS, " Pindar and the afterlife ", dans ID., Greek Epic, Lyric and Tragedy: The Academic Papers of Sir H. Lloyd-Jones, Oxford 1990, p. 80-109 [avec addenda ; $1^{\text {re }}$ publication 1985].

- Plutarque, De facie: A. Lernould (dir.), Plutarque. Le visage qui apparaît dans le disque de la Lune (De facie quae in orbe lunae apparet), texte grec, traduction, notes et trois études de synthèse, Villeneuve d'Ascq 2013. 
- Posidonius : K. Algra, "Posidonius d'Apamée ", dans R. Goulet (éd.), Dictionnaire des philosophes antiques, vol. Vb, Paris 2012, p. 1481-1499. * A. E. Ju, "Posidonius as historian of philosophy : an interpretation of Plutarch, De animae procreatione in Timaeo 22, 1023b-c ", dans M. Schofield (éd.), Aristotle, Plato and Pythagoreanism in the first century BC: new directions for philosophy, Cambridge 2013, p. 95-117.

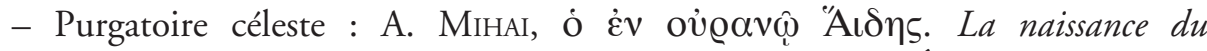
Purgatoire dans l'Antiquité, Thèse de Doctorat en co-tutelle, École Pratique des Hautes Études - Section des sciences religieuses, Paris et université de Montréal 2013 (dir. Ph. Hoffmann \& P. Bonnechere).

- Religion grecque : W. BURKERT, La religion grecque à l'époque archä̈que et classique, trad. et mise à jour bibliographique P. Bonnechere, Paris 2011.

- Rites funéraires: Thesaurus Cultus et Rituum Antiquorum, vol. 1-8, Los Angeles 2004-2012, passim ; en partic. ThesCRA 6 (2011), 1.e : "Mort et inhumation » + 8 (2012), addendum, p. 363-384 (V. VLACHOU).

- Soleil (théologie solaire) : P. BoyAncé, "L'Apollon solaire ", dans Mélanges d'archéologie, d'épigraphie et d'histoire offerts à Jérôme Carcopino, Paris 1966, p. 149-170. * W. Fauth, "Pythagoras, Jesus von Nazareth und der Helios-Apollon des Julianus Apostata : zu einigen Eigentümlichkeiten der spätantiken Pythagoras-Aretalogie im Vergleich mit der thaumasiologischen Tradition der Evangelien ", Zeitschrift für die Neutestamentliche Wissenschaft 68 (1987), p. 26-48. * ID., Helios Megistos : zur synkretistischen Theologie der Spätantike, Leiden 1995. * ID., "Salutatio solis orientis: zu einer Form der Heliolatrie bei Pythagoreern, Manichäern, Therapeuten und Essenern ", dans H. Cancik, H. Lichtenberger, P. Schäfer (éd.), Geschichte-Tradition-Reflexion : Festschrift für Martin Hengel zum 70. Geburtstag, vol. 2. Griechische und römische Religion, Tübingen 1996, p. 41-54.

- Songe de Scipion : N. LÉVI, "Arcana in ipso consummati operis fastigio " : la révélation finale dans la littérature latine (Cicéron, Ovide, Apulée), Thèse de doctorat, Université Paris-Sorbonne 2011 (dir. C. Lévy).

- Sotériologie : V. Adluri (éd.), Philosophy and salvation in Greek religion, Berlin 2013. * I. Chirassi Colombo, "La salvezza nel aldilà nella cultura greca arcaica ", Studii Clasice 15 (1973), p. 23-39. * R. Gordon, "Authority, salvation and mystery in the mysteries of Mithras ", dans J. Huskinson, M. Beard, J. Reynolds (éd.), Image and mystery in the Roman world : three papers given in memory of Jocelyn Toynbee, Cambridge 1988, p. 45-80. * Pagani e cristiani alla ricerca della salvezza (secoli I-III). XXXIV incontro di studiosi dell'antichità cristiana, Roma, 5-7 maggio 2005, Roma 2006. * G. Sfameni Gasparro (éd.), Destino e salvezza, tra culti pagani e gnosi cristiana: itinerari storico-religiosi sulle orme di Ugo Bianchi, Cosenza 1998.

- Symbolisme funéraire des sarcophages : J. Elsner, J. Huskinson (éd.), Life, death and representation: some new work on Roman sarcophagi, Berlin-New York 2011. * R. TuRCAN, Messages d'outre-tombe : l'iconographie des sarcophages romains, Paris 1999. * ID., Études d'archéologie sépulcrale : sarcophages romains et gallo-romains, Paris 2003. 
- Transmigration des âmes et réincarnation : A. Bernabé, M. Kahle, M.A. Santamaría (éd.), Reencarnación. La transmigración de las almas entre Oriente y Occidente, Madrid 2011. * A. CASAnOva, " La critica di Diogene d'Enoanda alla metempsicosi Empedoclea (NF 2 + frg. 34 Ch.) ", dans Studi in onore di Adelmo Barigazzi, Roma 1984, vol. 1, p. 51-72. * Cl. Poulle, G. Ducoeur (éd.), La transmigration des âmes en Grèce et en Inde anciennes. Actes de la Journée d'étude de l'université de Franche Comté (Besançon, 17 octobre 2013), à paraitre en 2014 dans la revue Dialogues d'histoire ancienne. ${ }^{*}$ F. SOLMSEN, " Reincarnation in ancient and early Christian thought », dans ID., Kleine Schriften, vol. 3, Hildesheim / Zürich / New York 1982, p. 465-494.

- Virgile, Enéide VI : J.N. BREMmer, «The golden bough : Orphic, Eleusinian and Hellenistic-Jewish sources of Virgil's underworld in Aeneid VI ", Kernos 22 (2009), p. 183-208.

- Voyage céleste : A. Destro et M. Pesce, "Le voyage céleste : tradition d'un genre ou schéma culturel en contexte ?", dans N. Belayche et J.-D. Dubois (éd.), L'oiseau et le poisson: cohabitations religieuses dans les mondes grec et romain, Paris 2011, p. 359-384.

- Voyages dans l'au-delà : J. J. Collins, M. Fishbane (éd.), Death, ecstasy, and other worldly journeys, Albany (New York) 1995. * Cl. Kappler et al. (éd.), Apocalypses et voyages dans l'au-delà, Paris 1987.

COnSTANTINOS Macris

CNRS-LEM (UMR 8584)

7, rue Guy Môquet

F 94801 Villejuif cedex

macris@vjf.cnrs.fr 